\title{
Sequenciamento de Ações Pedagógicas por Algoritmo Genético Utilizando Taxonomia de Bloom e ASSIST
}

\author{
Newarney T. Costa ${ }^{1}$, Márcia A. Fernandes ${ }^{2}$ \\ ${ }^{1}$ Instituto Federal Goiano (IF Goiano) \\ Iporá - GO - Brasil \\ ${ }^{2}$ Faculdade de Computação - Universidade Federal de Uberlândia (UFU) \\ Uberlândia - MG - Brasil \\ newarney.costa@ifgoiano.edu.br, marcia@ufu.br
}

\begin{abstract}
This paper proposes the automatic and personalized sequencing of pedagogical actions, from the point of view of automated planning based on genetic algorithm. The actions were modeled from the structure presented by the two-dimensional analysis of the Bloom Taxonomy (TB). The personalization of the actions occurs in accordance with the cognitive profile of the student, guided by the characteristics given by Approaches and Study Skills Inventory for Students (ASSIST). For this purpose, a relationship between TB and ASSIST was mapped in this work. The results show that it is possible to perform the sequencing of personalized pedagogical actions based on the student's cognitive profile, from the proposed relationship.
\end{abstract}

Resumo. Este trabalho propõe o sequenciamento automático e personalizado de ações pedagógicas, sob a perspectiva do planejamento automatizado baseado em algoritmo genético. As ações foram modeladas a partir da estrutura apresentada pela análise bidimensional da Taxonomia de Bloom (TB). A personalização das ações ocorre em consonância com o perfil cognitivo do estudante, pautado nas características dadas pelo Approaches and Study Skills Inventory for Students (ASSIST). Para tal, neste trabalho, foi mapeada uma relação entre TB e o ASSIST. Os resultados mostram que é possível realizar o sequenciamento de ações pedagógicas personalizadas, tendo como base o perfil cognitivo do estudante, a partir da relação proposta.

\section{Introdução}

O sequenciamento personalizado e automatizado de ações pedagógicas com intuito de otimizar o processo de aprendizagem, frequentemente conta com técnicas de Inteligência Artificial (IA). Dentre essas técnicas, o Planejamento Automatizado (PA) se destaca, pois consiste na seleção de ações para atingir um objetivo. Nesse processo, espera-se que o conjunto de ações escolhidas seja otimizado [Costa et al. 2019a].

Nos últimos anos, observa-se a evolução de trabalhos que utilizam técnicas de PA com o propósito supracitado. Entretanto, nota-se que a Planning Domain Definition Language (PDDL), linguagem comumente utilizada para o PA, pode não ser uma opção viável no contexto de sequenciamento automático de ações pedagógicas, pois à medida que a caracterização do estudante é refinada e são utilizados mais parâmetros para auxiliar 
no planejamento, tornam-se mais limitadas as heurísticas que possibilitam a busca no espaço de soluções [Costa et al. 2019b].

O uso de algoritmos baseados em computação evolutiva como apoio ao PA para realização de sequenciamento de ações pedagógicas, parte do princípio de que esse tipo de problema pode ser interpretado como um problema de otimização [Hssina and Erritali 2019]. Diante disso, a grande disponibilidade de dados justifica o uso dessa técnica, pois a manipulação destes por meio de outras técnicas, como o PA via PDDL por exemplo, exigiriam alto esforço computacional, inviabilizando, portanto, tal aplicação [Ariyaratne and Fernando 2014].

A modelagem das ações pedagógicas é outro fator decisivo no processo de sequenciamento. Nesse sentido, ações genéricas mostram-se interessantes na melhoria do processo de aprendizagem, uma vez que o foco desse sequenciamento passa a ser o processo cognitivo do estudante, em detrimento de um conteúdo específico. Assim, a Taxonomia de Bloom (TB) se apresenta como uma alternativa viável, pois estrutura tais ações a partir do domínio cognitivo do estudante [Krathwohl 2002].

A observação de características cognitivas do estudante são fundamentais para o sucesso no processo de personalização do sequenciamento pedagógico. As preferências, modeladas por meio de estilos de aprendizagem, são utilizadas em [Garrido et al. 2016]. Metadados de Ambiente Virtual de Aprendizagem (AVA) também podem ser utilizados com esse propósito, como observado em [Pireva and Kefalas 2018]. Já em [Limongelli and Sciarrone 2014], o Estado Cognitivo (EC) do estudante, representado por meio da Taxonomia de Bloom, é utilizado. Além dessas características, outras que podem ser utilizadas individualmente ou combinadas, de modo a compor um conjunto de atributos do estudante. Uma alternativa explorada para recomendações pedagógicas pode ser observada em [Costa et al. 2019a], onde o Approaches and Study Skills Inventory for Students (ASSIST) é utilizado para compor o perfil cognitivo do estudante no processo de recomendação de ações pedagógicas.

Diante do cenário exposto, este trabalho propõe o sequenciamento de ações pedagógicas estruturadas sob a perspectiva da Taxonomia de Bloom. Esse sequenciamento ocorre usando planejamento automatizado, implementado por meio de algoritmo genético (AG). Além disso, o perfil do estudante é caracterizado pelos atributos fornecidos pelo ASSIST.

Na Seção 2, é apresentada a revisão da literatura e estabelecida a relação entre a TB e o ASSIST. Na Seção 3, é abordada a proposta desse trabalho, incluindo os resultados obtidos. Na Seção 4, é feita uma explanação acerca das considerações finais, que versam sobre pontos positivos e negativos deste trabalho, além das perspectivas de desdobramentos a partir desta proposta.

\section{Revisão da Literatura}

Para a realização do processo de sequenciamento de ações pedagógicas, é necessária a ponderação de diversos elementos, como o planejador adequado para esse sequenciamento, por exemplo. Além disso, a modelagem das ações e a definição das características do estudante podem ser fundamentais para o sucesso da abordagem. A partir desse cenário, tais questões são discutidas nas próximas subseções. 
IX Congresso Brasileiro de Informática na Educação (CBIE 2020)

Anais do XXXI Simpósio Brasileiro de Informática na Educação (SBIE 2020)

\subsection{Planejamento Automatizado Apoiado por Computação Evolutiva}

O uso de algoritmos evolutivos para realização do planejamento automatizado é viável no contexto de sequenciamento pedagógico. Sob o ponto de vista do perfilamento do estudante, distintas características podem ser abordadas. Por exemplo, os estilos de aprendizagem são explorados nos trabalhos apresentados em [Machado et al. 2018]. Além disso, as preferências também compõem a modelagem do estudante em [Lin et al. 2016, de Miranda et al. 2019]. O nível de conhecimento como premissa para recomendação pedagógica é adotado em [Hssina and Erritali 2019, de Miranda et al. 2019]. Já em [Vanitha et al. 2019], observa-se que aspectos como objetivos de aprendizagem, estado emocional, habilidade cognitiva e desempenho do estudante foram utilizados na modelagem.

Dentre as abordagens evolutivas, o algoritmo de otimização de colônia de formigas foi explorado em [Rastegarmoghadam and Ziarati 2017, Vanitha et al. 2019]. Já o Algoritmo Genético é explorado em [Lin et al. 2016, Hssina and Erritali 2019, de Miranda et al. 2019, Vanitha et al. 2019]. Além dessas técnicas, o Algoritmo PresaPredador é abordado em [Machado et al. 2018]. Nesses trabalhos, a abordagem evolutiva é utilizada sob a perspectiva de problemas de otimização no contexto de sequenciamento de ações pedagógicas.

Os problemas de otimização abordados no cenário de aprendizagem são diversos, dentre os quais podemos destacar: i) A busca pela rota de aprendizagem ideal, de acordo com o perfil do estudante, observada em [Rastegarmoghadam and Ziarati 2017, Hssina and Erritali 2019, Vanitha et al. 2019]; ii) o desempenho do estudante e o tempo de aprendizagem, abordados em [Lin et al. 2016]; iii) o sequenciamento curricular, como visto em [Agbonifo and Olanrewaju 2018]; iv) a satisfação do estudante, além da minimização e equalização no tempo de trabalho entre os participantes de um grupo são tratados em [de Miranda et al. 2019].

Diante dos trabalhos supracitados, constata-se que o sequenciamento de ações pedagógicas pode ser interpretado como um problema de otimização. Sob essa ótica, é viável a utilização de algoritmos evolutivos na busca soluções para esse tipo de problema [Hssina and Erritali 2019]. Nesse cenário, a grande disponibilidade de dados justifica o uso da estratégia supracitada, pois a manipulação desses por meio de técnicas como o PA via PDDL, por exemplo, exigiriam alto esforço computacional e inviabilizariam tal aplicação [Ariyaratne and Fernando 2014]. Ainda nesse contexto, nota-se a viabilidade de utilização do AG como técnica para apoiar o PA com o intuito de realizar o sequenciamento de ações pedagógicas.

\subsection{Modelagem de Ações Pedagógicas Utilizando Taxonomia de Bloom}

No trabalho apresentado em [Costa et al. 2019a], a recomendação de ações pedagógicas pautadas no processo cognitivo do estudante, pode trazer benefícios, pois não depende da estruturação curricular de um curso específico, por exemplo. Assim, a estruturação de ações pedagógicas segundo a Taxonomia Bloom (TB) revisada em [Krathwohl 2002], pode ser uma alternativa. A TB apresenta uma análise bidimensional a partir da dimensão do processo cognitivo (DPC) e da dimensão do conhecimento (DC) e pode auxiliar no planejamento didático-pedagógico. 
Na TB, a DPC é composta por seis estados cognitivos (ECs) hierarquicamente estruturados a partir de Lower Order Cognitive Skills (LOCS), para Higher Order Cognitive Skills (HOCS). Cada um desses estados é representado por um conjunto de ações que podem ser desenvolvidas pelo estudante no contexto de aprendizagem. Já a DC, Diz respeito ao conhecimento adquirido ou produzido pelo estudante e é estruturada em quatro níveis a partir do nível mais concreto, para o nível mais abstrato [Krathwohl 2002]. A Figura 1 apresenta a estruturação das ações pedagógicas sob a ótica da análise bidimensional proposta pela TB.

\begin{tabular}{|c|c|c|c|c|c|c|c|}
\hline & & \multicolumn{6}{|l|}{ LOCS } \\
\hline & $\mathrm{DC} \quad \mathrm{DPC}$ & Lembrar & $\begin{array}{c}\text { Compre- } \\
\text { ender }\end{array}$ & Aplicar & Analisar & Avaliar & Criar \\
\hline CONCRETO & Factual & ação 1 & ação 5 & ação 9 & ação 13 & ação 17 & ação 21 \\
\hline \multirow{2}{*}{$\begin{array}{l}\uparrow \\
\vdots \\
\forall\end{array}$} & Conceitual & ação 2 & ação 6 & ação 10 & ação 14 & ação 18 & ação 22 \\
\hline & Procedural & ação 3 & ação 7 & ação 11 & ação 15 & ação 19 & ação 23 \\
\hline ABSTRATO & Metacognitivo & ação 4 & ação 8 & ação 12 & ação 16 & ação 20 & ação 24 \\
\hline
\end{tabular}

Figura 1. Estruturação de ações pedagógicas na Taxonomia de Bloom.

Segundo [Krathwohl 2002], é observada a possibilidade de flexibilização na hierarquia da $\mathrm{TB}$, em detrimento da percepção do perfil cognitivo do estudante. Logo, as possibilidades de arranjos nas sequências de ações pedagógicas são de de 16.777.216, pois na abordagem bidimensional da TB a quantidade total de ações é 24, como mostrado na Figura 1.

Diante do cenário exposto, uma sequência de ações possível, seria: $\operatorname{seq} 1=$ $[1,2,4,5,6,7,8,15,17,18,19,20,22,24]$. Note que pela definição da TB, não necessariamente todas as ações precisam ser utilizadas, porém as restrições hierárquicas LOCS/HOCS e concreto/abstrato devem ser respeitadas.

\subsection{Caracterização de Estudantes Usando Perfil Cognitivo ASSIST}

O ASSIST apresentado em [Entwistle and Tait 2013], utiliza uma dimensão de análise do perfil cognitivo do estudante, a partir do Revised Approaches to Studying Inventory (RASI), que é composto por um questionário com 52 itens. Nessa abordagem, o perfil cognitivo do estudante é dado por três eixos e cada um desses é composto por subcategorias que os descrevem, conforme apresentadas na Tabela 1.

Tabela 1. Perfis cognitivos ASSIST e suas subescalas.

\begin{tabular}{|l|l|l|}
\hline \multicolumn{1}{|c|}{ Surface } & \multicolumn{1}{c|}{ Strategic } & \multicolumn{1}{c|}{ Deep } \\
\hline - Falta de propósito. & - Estudo organizado. & - Busca por significado. \\
- Memorização não relacio- & - Gerenciamento de tempo. & - Relacionamento de ideias. \\
nada. & - Realização. & - Uso de evidência. \\
- Medo do fracasso. & - Prontidão para demandas & - Interesse em ideias. \\
- Limite do plano de estudos. & de avaliação. & - Monitoramento da eficácia. \\
\hline
\end{tabular}
Fonte: Adaptado de [Entwistle and Tait 2013].

O ASSIST proporciona identificar o perfil cognitivo do estudante (ASSIST_E〈su,st,de $\rangle$ ) em função dos índices obtidos para os eixos surface 
(su), strategic (st) e deep (de). Um exemplo para essa classificação poderia ser: $A S S I S T \_E($ estudante $A)\langle s u, s t, d e\rangle=\langle 0.34375,0.5,0.65\rangle$, onde cada índice indica a relevância de cada eixo para o perfil cognitivo do estudante. Cada um dos perfis cognitivos no ASSIST apresenta uma variação em relação às habilidades do estudante, variando de LOCS para HOCS [Entwistle et al. 1997].

\subsection{Mapeamento da Relação entre ASSIST e TB}

Conforme discutido nas subseções 2.2 e 2.3, tanto a TB quanto o ASSIST, apresentam uma evolução em relação ao estado cognitivo do estudante, variando de LOCS para HOCS. Tais teorias apresentam um ponto de convergência ao explorar a representação do processo cognitivo considerando um espectro evolutivo partindo de elementos concretos para abstratos. Nos trabalhos apresentados em [Brown et al. 2015, Shang 2019], é explorada a relação entre os perfis cognitivos definidos pelo ASSIST e as categorias da DPC apresentadas na TB.

Diante das considerações supracitadas, observa-se a possibilidade de mapeamento entre os perfis cognitivos ASSIST e a TB. Neste trabalho realizou-se esse mapeamento, classificando cada uma das 52 questões dadas pelo ASSIST, de acordo com sua aderência aos estados cognitivos definidos pela TB. Para tal, foram utilizados os verbos-chave que compõem cada um dos ECs na TB. Na Tabela 2 a relação mapeada é mostrada e os pesos apresentados expressam os índices ASSIST associados entre a TB e os perfis cognitivos.

Tabela 2. Estruturação de ações pedagógicas na Taxonomia de Bloom.

\begin{tabular}{|l|c|c|c|c|c|c|}
\hline ESSIST & $\begin{array}{c}\text { Lembrar } \\
\left(P R_{1}\right)\end{array}$ & $\begin{array}{c}\text { Compreender } \\
\left(P R_{2}\right)\end{array}$ & $\begin{array}{c}\text { Aplicar } \\
\left(P R_{3}\right)\end{array}$ & $\begin{array}{c}\text { Analisar } \\
\left(P R_{4}\right)\end{array}$ & $\begin{array}{c}\text { Avaliar } \\
\left(P R_{5}\right)\end{array}$ & $\begin{array}{c}\text { Criar } \\
\left(P R_{6}\right)\end{array}$ \\
\hline Surface $(s u)$ & 0.6250 & 0.125 & 0.0000 & 0.125 & 0.0000 & 0.1250 \\
\hline Strategic $(s t)$ & 0.0625 & 0.125 & 0.1875 & 0.250 & 0.3125 & 0.0625 \\
\hline Deep $(d e)$ & 0.0500 & 0.150 & 0.0000 & 0.350 & 0.3000 & 0.1500 \\
\hline
\end{tabular}

Considerando que uma sequência de ações pode ser definida conforme estrutura apresentada na Figura 1, o cálculo dos índices ASSIST para essa sequência ( $A S S I S T \_A\langle s u, s t, d e\rangle$ ) é dado pelo somatório do produto entre $P R$ e $P S$ em cada EC. $P R$ é dado pela relação descrita na Tabela 2. $P S$ é dado pela soma da quantidade de ações em cada EC, dividida por 4. Então, $P S \in[0,1]$, pois a quantidade máxima de ações por EC é 4. Assim, na sequência de ações dada como exemplo na Subseção 2.2, temos: $P S($ seq 1$)=[0.75,1.0,0,0.25,1.0,0,5]$. Logo, o somatório $P R_{1} * P S_{1}+\ldots+P R_{6} * P S_{6}$ para cada um dos índices ASSIST na Tabela 2 produz o seguinte resultado: $A S S I S T \_A(s e q 1)\langle s u, s t, d e\rangle=\langle 0.6875,0.578125,0.65\rangle$. Deste modo, temos os índices ASSIST surface, strategic e deep de uma sequência de ações.

\section{Modelo Proposto}

A partir do contexto discutido na Seção 2, o planejamento automatizado, apoiado por algoritmo genético pode ser uma alternativa viável para a recomendação de ações pedagógicas personalizadas. Assim, neste trabalho, tais recursos são utilizados para sequenciar ações pedagógicas modeladas pela Taxonomia de Bloom, personalizadas de acordo com o perfil cognitivo do estudante. 
IX Congresso Brasileiro de Informática na Educação (CBIE 2020)

Anais do XXXI Simpósio Brasileiro de Informática na Educação (SBIE 2020)

\subsection{Sequenciamento de Ações Pedagógicas Usando AG}

Neste trabalho, a modelagem das ações pedagógicas é realizada segundo a Taxonomia de Bloom, discutida na Subseção 2.2. Além disso, tal modelagem faz uso das condições oferecidas pelo PA, pois o sequenciamento de ações pedagógicas leva em consideração um objetivo pedagógico a ser alcançado. Desse modo, o estado inicial do planejamento é a identificação do perfil cognitivo do estudante e o objetivo desse planejamento é encontrar uma sequência de ações aderente a esse perfil.

A implementação do AG, neste trabalho, considerou os parâmetros estabelecidos em [Engelbrecht 2007]. Assim, foi criada uma população inicial, em seguida os indivíduos dessa população foram avaliados até que o critério de parada fosse atingido. Durante esse processo, os operadores genéticos de mutação e de cruzamento também foram adotados, conforme detalhes discutidos a seguir.

O indivíduo do AG proposto é composto por uma sequência de 24 bits, lidos da esquerda para a direita. Estes representam cada uma das possíveis ações da TB, considerando sua análise bidimensional. Portanto, o primeiro bit representa a ação 1 , o segundo bit a ação 2 e assim por diante, até o $24^{\circ}$ bit, que representa a ação 24 , conforme as ações estruturadas na Figura 1. Quando o valor de um bit for 1, significa que aquela ação fará parte do sequenciamento e quando esse valor for zero 0 , tal ação não estará presente no sequenciamento. Observa-se também que o indivíduo tem tamanho fixo de 24 bits, porém a sequência de ações pedagógicas tem tamanho variável, pois apenas os bits com valor 1 representam as ações sequenciadas. Um exemplo do indivíduo é mostrado na Figura 2.

\begin{tabular}{|c|c|c|c|c|c|c|c|c|c|c|c|c|}
\hline TB & \multicolumn{4}{|c|}{ Lembrar } & \multicolumn{4}{|c|}{ Compreender } & \multicolumn{4}{|c|}{ Aplicar } \\
\hline Posição & $\overline{10}$ & $2^{\circ}$ & $3^{0}$ & $\overline{4^{0}}$ & $5^{0}$ & $6^{0}$ & $7^{0}$ & $8^{\circ}$ & $9^{\circ}$ & $10^{\circ}$ & $11^{\circ}$ & $12^{\circ}$ \\
\hline Valor & 1 & 1 & 0 & 1 & 1 & 0 & 0 & 1 & 0 & 0 & 1 & 1 \\
\hline TB & \multicolumn{4}{|c|}{ Analisar } & \multicolumn{4}{|c|}{ Avaliar } & \multicolumn{4}{|c|}{ Criar } \\
\hline Posição & $13^{\circ}$ & $14^{\circ}$ & $15^{\circ}$ & $16^{\circ}$ & $17^{\circ}$ & $18^{\circ}$ & $19^{\circ}$ & $20^{\circ}$ & $21^{\circ}$ & $22^{\circ}$ & $23^{\circ}$ & $24^{\circ}$ \\
\hline Valor & 1 & 0 & 0 & 1 & 0 & 0 & 1 & 1 & 0 & 0 & 1 & 1 \\
\hline
\end{tabular}

Figura 2. Representação binária do indivíduo no AG.

A modelagem do indivíduo, conforme apresentada na Figura 2, sempre gera uma sequência de ações válidas. Note que na TB, a hierarquia dos estados cognitivos pode ser adequada às necessidades do estudante [Krathwohl 2002]. Nessa hierarquia, é desejável que ações pedagógicas menos complexas, ou seja, nas posições do indivíduo cujos bits com valor 1 estejam mais à esquerda, ocorram antes de atividades mais complexas, ou seja, os bits com valor 1 cujas posições estejam mais à direita do indivíduo. Desse modo, a sequência de ações pedagógicas, seguindo a ordem da esquerda para a direita, sempre respeitará tal restrição.

Na primeira etapa do AG proposto, é criada a população com a quantidade $m a x \_i$ de indivíduos que representam potenciais soluções. Estes são gerados aleatoriamente e codificados em uma matriz binária, onde cada linha possui 24 bits. Cada bit de um indivíduo representa uma ação de acordo com a estrutura apresentada na Figura 2. A função de aptidão (fintess), dada pela Eq. 1, é calculada em relação aos índices ASSIST do indivíduo $(I)$ e do estudante $(E)$. O problema então consiste em minimizar essa função, 
que é a distância euclidiana entre os índices ASSIST de $I$ e $E$, acrescida de uma penalidade, caso os critérios de relevância entre esses índices não sejam satisfeitos. A distância euclidiana é dada pela Eq. 2, onde $s u$, st e de representam os índices dos perfis ASSIST surface, strategic e deep, respectivamente e são obtidos conforme descritos nas subseções 2.3 e 2.4 .

$$
\text { fitness }(I)=\operatorname{distancia}(I, E)+\text { penalidade }(I, E)
$$

$$
\operatorname{distancia}(I, E)=\sqrt{(s u(I)-s u(E))^{2}+(s t(I)-s t(E))^{2}+(\operatorname{de}(I)-\operatorname{de}(E))^{2}}
$$

A penalidade $(I, E)$ é calculada considerando a comparação da ordem de relevância dos índices ASSIST entre $I$ e $E$. A cada índice é atribuído um peso 3, 2 ou 1 , conforme a relevância de cada índice, sendo 3 para o mais relevante e 1 para o menos. Desse modo a penalidade é a soma do resultado de (distancia $(I, E) / 6 *$ peso) para cada um dos índices com relevância discrepante. Utilizando o perfil de estudante e a sequência de ações dados como exemplos nas subseções 2.3 e 2.4, temos a ordem de relevância mostrada na Tabela 3. Nesse exemplo, a penalidade é calculada considerando os

\begin{tabular}{|c|c|c|c|c|c|c|}
\hline & \multicolumn{3}{|c|}{ Índices ASSIST } & \multicolumn{3}{|c|}{ Pesos / Ordem de relevância } \\
\hline & su & $s t$ & de & Peso 3 & Peso 2 & Peso 1 \\
\hline Indivíduo $(I)$ & 0.687500 & 0.578125 & 0.65000 & su & $d e$ & $s t$ \\
\hline Estudante $(E)$ & 0.343750 & 0.500000 & 0.65000 & de & $s t$ & su \\
\hline
\end{tabular}

Tabela 3. Exemplo da ordem de relevância dos índices ASSIST.

pesos 3,2 e 1, pois a ordem de relevância para todos os índices é divergente. Logo temos: penalidade $(I, E)=\operatorname{distancia}(I, E) / 6 * 3+\operatorname{distancia}(I, E) / 6 * 2+\operatorname{distancia}(I, E) / 6$. A partir disso, exemplos de cálculos para distancia, penalidade e fitness são:

- $\operatorname{distancia}(I, E)=\sqrt{(0.6875-0.34375)^{2}+(0.578125-0.5)^{2}+(0.65-0.65)^{2}}=0.352516$

- penalidade $(I, E)=0.352516 / 6 * 3+0.352516 / 6 * 2+0.352516 / 6=0.352516$

- $\operatorname{fitness}(I)=\operatorname{distancia}(I, E)+$ penalidade $(I, E)=0.705032$

Note que a penalidade pode, no máximo, dobrar o valor dado pela a distância euclidiana, como nesse exemplo, em que todos os índices ASSIST apresentam relevância divergente.

O critério de parada do AG ocorre quando o número máximo de gerações $\left(\max _{-} g\right.$ ) é atingido ou o fitness do indivíduo tem o valor zero. A seleção dos indivíduos que comporão a próxima geração da população é obtida por meio de torneio. Para isso, é feita a seleção aleatória de três indivíduos da população, optando pela escolha do indivíduo mais apto, segundo uma probabilidade $k_{\_} t$. Essa abordagem pode reduzir o peso dos indivíduos melhor avaliados para o algoritmo e proporcionar maior diversidade à população.

O cruzamento no AG é realizado considerando blocos de 4 bits, dadas as 6 dimensões da TB que estes blocos representam. A quantidade de blocos e quais os blocos serão permutados são determinados aleatoriamente. Após essa etapa, caso o fitness do novo indivíduo seja melhor do que pelo menos um dos indivíduos que o geraram, este torna-se apto a compor o ranqueamento para formação da nova população. Caso contrário, o novo indivíduo é descartado. No ranqueamento, são selecionados os $\max \_i$ 
IX Congresso Brasileiro de Informática na Educação (CBIE 2020)

Anais do XXXI Simpósio Brasileiro de Informática na Educação (SBIE 2020)

indivíduos mais aptos para fazerem parte da nova população, dentre a população atual e os novos indivíduos gerados pelo cruzamento. Após a geração da nova população, é realizada a mutação com taxa $k_{-} m$ de indivíduos da população. A mutação realiza a inversão aleatória de 1 bit de cada bloco.

\subsection{Resultados Obtidos}

O experimento realizado teve o objetivo de selecionar automaticamente uma sequência de ações pedagógicas cujos índices ASSIST fossem o mais próximos possíveis ao perfil cognitivo do estudante. Para validação de viabilidade do AG implementado, foram identificados 17 perfis cognitivos de estudantes do ensino superior, na área de Computação, por meio da coleta de respostas ao questionário ASSIST.

Para o AG proposto, foram definidos o tamanho da população $\max _{-} i=1000$ e o número de gerações $\max _{-} g=100$. Na seleção de indivíduos por torneio, a probabilidade $k \_t$ foi de $60 \%$ e a probabilidade de mutação $k \_m$, foi de $10 \%$. Testes exploratórios foram realizados com a finalidade de averiguar os impactos da alteração desses parâmetros na eficácia do AG, porém as diferenças nos resultados não foram significativas ou pioraram os resultados. Para cada um dos estudantes, o AG foi executado 30 vezes. A

Tabela 4. Fitness das sequências de ações mais aderentes os perfis cognitivos.

\begin{tabular}{|l|c|c|c|}
\hline Fitness & $\leq \mathbf{0 . 0 5}$ & $>\mathbf{0 . 0 5}$ e $\leq \mathbf{0 . 1}$ & $>\mathbf{0 . 1}$ \\
\hline Perfis cognitivos & 14 & 2 & 1 \\
\hline
\end{tabular}

média do fitness nas 30 execuções para cada estudante é apresentada na Tabela 4. Para 14 indivíduos esta média foi $\leq 0.05$, significando que a taxa de compatibilidade com os perfis dos estudantes é acima de $97.5 \%$. Dentre esses, um indivíduo teve taxa de $100 \%$ de compatibilidade. Além disso, para apenas um estudante essa média foi 0.262 , ou seja, a taxa de compatibilidade foi de $84,87 \%$. A partir desses resultados, foi possível constatar a viabilidade do método proposto para realizar o sequenciamento automático de ações pedagógicas compatíveis com perfis ASSIST de estudantes.

\section{Considerações Finais}

O sequenciamento de ações pedagógicas é uma questão emergente no contexto educacional. Nota-se a importância de explorar técnicas que tenham como foco o processo cognitivo do estudante, para além de restrições curriculares. Assim, a modelagem de ações sob a perspectiva da Taxonomia de Bloom é interessante, pois essas são sistematizadas sob a ótica cognitiva do estudante. Além disso, a utilização do ASSIST para a modelagem do perfil cognitivo do estudante mostra-se pertinente, pois torna viável o estabelecimento de uma relação entre tal perfil e as ações pedagógicas estabelecidas pela TB.

Nota-se a praticabilidade da relação supracitada, pois em ambas as bases teóricas, o processo cognitivo do estudante é hierarquizado sob a perspectiva de variação no sentido de LOCS para HOCS. Desse modo, uma das contribuições deste trabalho é o complemento dessa relação a partir dos trabalhos de [Brown et al. 2015] e [Shang 2019], por meio do mapeamento entre a abordagem bidimensional da TB e o ASSIST, com base em análise das ações presentes na TB e os perfis cognitivos dados pelo questionário ASSIST. 
O planejamento automatizado tem como premissa o sequenciamento de ações para atingir um objetivo. No contexto de recomendações pedagógicas, sua implementação a partir da linguagem PDDL é interessante, pois permite a modelagem de ações bem definidas. Entretanto, constata-se que tal abordagem apresenta limitações à medida que as características do estudante são refinadas, tornando impraticáveis computacionalmente as heurísticas necessárias à busca no espaço de soluções. Uma alternativa adequada a esse problema é o PA apoiado por algoritmo genético.

Outra contribuição deste trabalho é a realização do sequenciamento de ações pedagógicas modeladas a partir do processo cognitivo do estudante, por meio de planejamento automatizado apoiado por algoritmo genético. Neste trabalho é possível notar a viabilidade na realização de sequenciamentos personalizados a partir da similaridade entre o perfil cognitivo do estudante dado pelo ASSIST e sequências de ações pedagógicas modeladas pela TB. Uma limitação deste trabalho, a ser explorada em trabalhos futuros, é incluir no perfil do estudante outras características, como nível de conhecimento e metadados de AVA, por exemplo. Como consequência, outras funções de aptidão poderão ser desenvolvidas.

A partir das implicações dos resultados deste trabalho, como proposta futura, objetiva-se a comparação do algoritmo genético com outras técnicas de computação evolutiva, afim de avaliar a efetividade entre tais técnicas. Outra proposta futura é a implementação da seleção de atividades de acordo com a Taxonomia Digital de Bloom, a fim de recomendá-las a estudantes em ambiente real, para tal, far-se-á necessário o mapeamento dessas atividades de acordo com a análise bidimensional da TB.

\section{Agradecimentos}

Os autores agradecem à Universidade Federal de Uberlândia (UFU) e ao Instituto Federal de Educação, Ciência e Tecnologia Goiano (IF Goiano) pelo apoio à esta pesquisa.

\section{Referências}

Agbonifo, O. C. and Olanrewaju, A. O. (2018). Genetic algorithm-based curriculum sequencing model for personalized e-learning system [j]. International Journal of Education and Management Engineering, 5(8):27-35.

Ariyaratne, M. and Fernando, T. (2014). A comparative study on nature inspired algorithms with firefly algorithm. International Journal of Engineering and Technology, 4(10):611-617.

Brown, S., White, S., Wakeling, L., and Naiker, M. (2015). Approaches and study skills inventory for students (assist) in an introductory course in chemistry. Journal of University Teaching \& Learning Practice.

Costa, N., Júnior, C. P., and Fernandes, M. (2019a). Recomendação de ações pedagógicas utilizando planejamento automático e taxonomia digital de bloom. In Brazilian Symposium on Computers in Education (Simpósio Brasileiro de Informática na EducaçãoSBIE), volume 30, page 1531.

Costa, N., Pereira Junior, C., Araújo, R., and Fernandes, M. (2019b). Application of ai planning in the context of e-learning. In International Conference on Advanced Learning Technologies (ICALT), page 57. 
IX Congresso Brasileiro de Informática na Educação (CBIE 2020)

Anais do XXXI Simpósio Brasileiro de Informática na Educação (SBIE 2020)

de Miranda, P. B., Ferreira, R., Castro, M. S., Neto, G. F., Souza, S. J., Santos, L. A., and Silva, L. L. (2019). Uma abordagem multiobjetivo para recomendação de caminhos de aprendizagem para grupo de usuários. Revista Brasileira de Informática na Educação, 27(3).

Engelbrecht, A. P. (2007). Computational intelligence: an introduction. John Wiley \& Sons.

Entwistle, N., McCune, V., and Tait, H. (1997). The approaches and study skills inventory for students (assist). Edinburgh: Centre for Research on Learning and Instruction, University of Edinburgh.

Entwistle, N. and Tait, H. (2013). Approaches and study skills inventory for students (assist) (incorporating the revised approaches to studying inventory - rasi). Edinburgh: Centre for Research on Learning and Instruction, University of Edinburgh.

Garrido, A., Morales, L., and Serina, I. (2016). On the use of case-based planning for e-learning personalization. Expert Systems with Applications, 60:1-15.

Hssina, B. and Erritali, M. (2019). A personalized pedagogical objectives based on a genetic algorithm in an adaptive learning system. Procedia Computer Science, 151:11521157.

Krathwohl, D. R. (2002). A revision of bloom's taxonomy: An overview. Theory Into Practice, 41(4):212-218.

Limongelli, C. and Sciarrone, F. (2014). Fuzzy student modeling for personalization of elearning courses. In Zaphiris, P. and Ioannou, A., editors, Learning and Collaboration Technologies. Designing and Developing Novel Learning Experiences, pages 292-301, Cham. Springer International Publishing.

Lin, Y.-S., Chang, Y.-C., and Chu, C.-P. (2016). An innovative approach to scheme learning map considering tradeoff multiple objectives. Journal of Educational Technology \& Society, 19(1):142-157.

Machado, M., Barrére, E., and Souza, J. (2018). Uma abordagem evolutiva para o problema de sequenciamento curricular adaptativo. In Brazilian Symposium on Computers in Education (Simpósio Brasileiro de Informática na Educação-SBIE), volume 29, page 1243.

Pireva, K. and Kefalas, P. (2018). A recommender system based on hierarchical clustering for cloud e-learning. Intelligent Distributed Computing XI, 53:235 - 245.

Rastegarmoghadam, M. and Ziarati, K. (2017). Improved modeling of intelligent tutoring systems using ant colony optimization. Education and Information Technologies, 22(3):1067-1087.

Shang, H. (2019). Cultural interpretation of deep approach to learning: an empirical analysis in a chinese university. In Cross-Cultural Business Conference 2019, page 207.

Vanitha, V., Krishnan, P., and Elakkiya, R. (2019). Collaborative optimization algorithm for learning path construction in e-learning. Computers \& Electrical Engineering, 77:325-338. 